\section{An inside view}

\section{Sean C. Solomon}

Planetary Interiors. By William B. Hubbard.

Van Nostrand Reinhold: 1984. Pp.334. $\$ 42.50, £ 49.75$.

THE United States planetary programme is midway through a five-year hiatus in major new observations from spacecraft missions, and an increasing number of American scientists are filling the gap by writing textbooks and monographs on various aspects of planetary science. Hubbard's offering treats the physics and chemistry of planetary interiors, covering all the planets (save Pluto) and most of the larger satellites viewed to date by spacecraft. The text incorporates material up to mid-1983, including results from the Voyager Saturn flyby and Pioneer Venus, but not the Soviet Venera 13-16 missions to Venus. A background in physics, chemistry, astronomy and geology at the level of a senior undergraduate or first-year graduate student is assumed.

The author has attempted a laudably comprehensive treatment of his subject. Discussion of the bulk composition and internal differentiation of each planet is cast in the framework of the composition of the Sun and the evolution of the solar nebula. Particularly thorough chapters are included on equations of state and planetary gravitational fields, both topics on which the author has made a number of original contributions. Accounts of the energy budgets of planetary interiors and the nature of planetary magnetic fields complete the foundation for the final half of the book, a thorough and quantitative inside tour through most of the principal objects of the Solar System.

Hubbard admits at the outset that "a single author has difficulty in writing a current, comprehensive book" on the planets. As if to prove the truth of this assertion, variations in the depth and evenness of coverage betray the background and research interests of the author. There is an implicit bias towards the outer planets, which first becomes apparent in the discussion on equations of state. The only silicate treated is "rock", defined as "that component of [nebular] condensate which is left after formation of an iron core". A chapter on the interior of the Earth is exposed as perfunctory with the opening apology that "this book is about the interiors of other planets"; worse, it contains misleading or incorrect statements about mantle convection, the nature of seismic discontinuities in the upper mantle and the evidence for a solid inner core.

There is a parallel bias towards an astrophysical, rather than a geological, approach to the planets. One telling statistic is that there is only a single photograph of a planetary or satellite surface (of the population genetics. His treatment of these issues is simple and lucid and will be readily comprehensible to the general reader. Having outlined the basic biological issues, Professor Jacquard focuses on subtleties which are sometimes ignored by advocates of a genetic basis to social policy and he tries to give a more balanced view of debatable ideas which are sometimes treated as "facts" in the popular mind. Among the problems he addresses are the "dysgenic" effects of medicine which, he argues, would take place extremely slowly even if they were not offset by such factors as genotype - environment interaction. His statement about consanguinity warrants some qualification. When writing of the origin of rules prohibiting consanguinity, he says (p.50), "the ill effects of consanguinity ... are much too slight to be observed empirically". It must be presumed that he is thinking about cousin marriage, since the effects of closer consanguinity on mental retardation, for example, are well documented.

The book points, indirectly, to the separation of population genetics from quantitative genetics which has characterized much of the development of both disciplines. Professor Jacquard's strength is in population genetics. When he turns to quantitative genetics and psychometrics he does not do justice to the best of either subject. For example, he invests great effort in criticizing an additive model for individual differences without asking why such a model often fits the data remarkably well. And he criticizes the concept of general intelligence without addressing the more interesting question of why multiple abilities are correlated.

Bertrand Russell observed that moral considerations could only appear when the truth is known and should not be used to decide what the truth is. There are few moral propositions in the book from which the sensitive reader is likely to dissent. Man's unalienable right to "life, liberty and the pursuit of happiness" is maintained, whether it be given by God, history or DNA. In spite of the growth of knowledge, we do not have all the facts to manipulate the future of the species in the broadest sense. Is this so bad? Professor Jacquard suggests that it is not. The title of his book reflects his belief, expressed in the final chapter, that human diversity, guaranteed by the mechanisms of inheritance and natural selection, is a treasure which should not be sacrificed before any idol of "progress" or "uniformity". This principle extends as much to the fruits of man's intellect as it does to the species itself. The dissemination of scientific ideas, and their open discussion divorced from political influences, is the best insurance that science will remain a guardian of freedom rather than a slave to totalitarianism.

Lindon J. Eaves is Distinguished Professor of Human Genetics at the Medical College of Virginia, Richmond, Virginia. 